\title{
GRAPHLET DATA MINING OF ENERGETICAL INTERACTION PATTERNS IN PROTEIN 3D STRUCTURES
}

\author{
Carsten Henneges, Marc Röttig \\ Center for Bioinformatics Tübingen, Eberhard Karls Universität Tübingen, Sand 1, Tübingen, Germany \\ Oliver Kohlbacher, Andreas Zell \\ Center for Bioinformatics Tübingen, Eberhard Karls Universität Tübingen, Sand 1, Tübingen, Germany
}

\begin{abstract}
Keywords: $\quad$ Graphlets, Data Mining, Relative Neighbourhood Graph, Secondary Structure Elements, Decision Tree Model Selection

Abstract: Interactions between secondary structure elements (SSEs) in the core of proteins are evolutionary conserved and define the overall fold of proteins. They can thus be used to classify protein families. Using a graph representation of SSE interactions and data mining techniques we identify overrepresented graphlets that can be used for protein classification. We find, in total, 627 significant graphlets within the ICGEB Protein Benchmark database (SCOP40mini) and the Super-Secondary Structure database (SSSDB). Based on graphlets, decision trees are able to predict the four SCOP levels and SSSDB (sub)motif classes with a mean Area Under Curve (AUC) better than 0.89 (5-fold CV). Regularized decision trees reveal that for each classification task about 20 graphlets suffice for reliable predictions. Graphlets composed of five secondary structure interactions are most informative. Finally, we find that graphlets can be predicted from secondary structure using decision trees (5-fold CV) with a Matthews Correlation Coefficient (MCC) reaching up to 0.7.
\end{abstract}

\section{INTRODUCTION}

Secondary structure elements (SSEs), $\beta$-sheets and $\alpha$-helices, are important building blocks of proteins and interactions between these SSEs stabilize protein tertiary structures. Many categorization schemes are based on these SSEs like the SCOP (Murzin et al., 1995) or CATH (Orengo et al., 1997) databases. For example, the SCOP categorization scheme has four levels, each one giving more detailed information about the SSEs of a protein domain and the arrangement of its SSEs.

An important question, especially in the context of SSE-based categorization schemes like SCOP, is whether there are interaction patterns between these SSEs that occur preferably in native protein structures or in protein structures of a given fold or superfamily. As SSEs are complex three-dimensional structures, whose relative states are hard to encode, analysing the SSE interactions in full atomic detail can become complex. However, encoding energetic interaction between SSEs as graphs makes this problem amenable for graph-based analysis of protein structures. The SSEs of a structure are mapped to vertices of a graph, and interactions between SSEs are represented by edges. Graph mining methods (Vacic et al., 2010) can then be applied on these reduced representations of protein structures.

In the following sections, we mine these graph representations for subgraphs, termed graphlets, that preferably occur in native protein structures. The significance of a graphlet is statistically tested using a random graph model, serving as a background model (Milo et al., 2002). This background model allows us to identify graphlets that occur more often in protein graphs than in random graphs. The value of the extracted graphlets is then demonstrated by using them in predictors for SCOP levels and SSSDB motifs.

\section{MATERIALS AND METHODS}

\section{Databases}

We perform our experiments using protein structures from the SCOP40mini dataset from the ICGEB database and the database of super-secondary struc-

190 
tures SSSDB (Sonego et al., 2007; Chiang et al., 2007).

\section{Protein Preparation}

We use $\mathrm{C}++$ routines and the BALL library (Kohlbacher and Lenhof, 2000) to analyse protein structures. As a first step, hydrogen atoms are added. After a consistency check of each residue, we assign partial charges and atom radii to atoms according to the AMBER force field. Finally, all hetero residues are removed from the structure.

To be independent from protein annotation, all SSEs are recomputed using the DSSP algorithm (Kabsch and Sander, 1983), afterwards all residues within loops are removed. $\alpha$-helices are not further modified, whereas all connected components of $\beta$ strands linked by hydrogen bonds are merged into $\beta$ sheets. In this way, each $\beta$-sheet is treated as a complete $3 \mathrm{D}$ structure during the computation of interaction energies.

\section{Graphical Encoding of Energetic SSE Interactions}

To prepare the graph construction, we compute the pairwise matrix of SSE interaction energies. Let $A$ and $B$ be any secondary structures in a protein, and let $E[A, B, \ldots]$ be the AMBER energy of a set of SSEs, then the pairwise interaction energy $I[A, B]$ is given as

$$
I[A, B]=E[A, B]-E[A]-E[B] .
$$

Graph construction serves for structural normalization as well as extracting the interaction model. It filters needless relations, while being independent from the computed amount of $I[A, B]$ energy and the relative distance of the SSEs. Therefore, it makes proteins having different numbers of SSEs comparable.

One convenient graph for this task is the Relative Neighbourhood Graph (RNG) (Toussaint, 1980). The RNG connects two labelled SSE nodes if the following edge condition

$$
I[A, B] \leq \max _{C}\{I[A, C], I[B, C]\},
$$

holds, where $A, B, C$ are SSEs from the protein and $A \neq B \neq C$. The RNG is a connected proximity graph and, therefore, also connects SSEs that are too distant for direct protein residue contacts. As its edge condition resembles an ultra-metric (Milligan and Isaac, 1980), the RNG has shown great robustness in practice and is a powerful tool to extract meaningful perceptual structures (Toussaint, 1980).

\section{Graphlet Analysis}

Graphlet analysis makes use of subgraph sampling and, therefore, relies on a graph isomorphism test. Each sampled subgraph is referred to as graphlet and its frequency or probability within a network is estimated by repeated sampling. In addition, statistical graphlet analysis requires the knowledge of a background distribution to compute the probability of an observation. As no analytical distribution function for graphlets is known, their probabilities are in general estimated from random graphs.

To obtain a random model resembling the input graph distribution, each protein graph is randomized. We use a random rewiring method where each edge is split into two half-edges. Then, all half-edges are randomized and rewired. This is repeated until a connected graph is obtained or a maximum number of iterations is reached. In the latter case, the last sample is saved. In summary, random rewiring conserves important graph properties (e.g. the node degree). By randomizing each graph once, we obtain a collection of random graphs that closely resembles the test distribution.

Next, we estimate the graphlet distribution by random sampling connected subgraphs. The goal of the sampling is twofold: First, all existent graphlets should be detected and, second, their distribution should be estimated correctly. If all graphlets were known in advance, drawing a fixed number of samples would yield the maximum likelihood estimate of the graphlet distribution, which is a multinomial distribution (Wassermann, 2004; Georgii, 2004). To achieve this estimate, we employ a two-pass approach for this task.

In the first pass, the data is exploratory sampled. For counting the graphlet frequencies, we make use of a Move-to-Front (MF) list that holds a counter for each graphlet type. Thus, each sampled graphlet is first searched in the MF list for counting its occurrence and inserted in the case it is not found. Therefore, the MF list length increases during this pass. We draw 1000 samples per graph of the database to minimize the possibility of missing patterns.

In the second pass, we keep the MF list fixed during sampling to compute the maximum likelihood estimates. Again, we draw a total of 1000 samples, 5 repetitions with 200 samples, from each graph and, thus, obtain 5 independent distribution estimates. If sampling detects an unknown graphlet within the second pass, a counter for unknown patterns is increased. Finally, we compute the distribution estimate by averaging and normalizing all samplings for a graph.

We choose a sampling size of 1000 graphlets in 
each phase because only a small fraction of the RNG comprises more than 40 nodes, i.e., only a small fraction of the database proteins are large. As the number of graphlets increases exponentially with pattern size, a trade-off between estimation accuracy and runtime is required. We found that a sampling size of 1000 is sufficient to accurately estimate the graphlet distribution within a reasonable runtime.

To determine significant graphlets, we employ permutation testing, which is a non-parametric technique to efficiently test the equality of two distributions $g, h$ (Wald and Wolfowitz, 1944). It requires no assumptions on the true probability distributions. By resampling new random distributions from the input distribution and evaluating a test statistic $T$, it infers the distribution of $T$ under $H_{0}: g=h$. This denotes the case where $g$ and $h$ are equal and, therefore, allows to compute the p-value for the hypothesis that the input distributions are equal. In our experiments, we use the coin package implemented in R (R Development Core Team, 2009).

For each graphlet, we employ permutation testing to compute the likelihood that its distribution on the protein graphs equals its distribution on the randomized graphs. Hence, we determine those graphlets that are significantly over or under-represented in random graphs. To account for the multiple-testing problem, we afterwards adjust the p-values according to the method of (Holm, 1979). Finally, all graphlets below a significance threshold of 0.05 and having a frequency ratio at least 10 -fold larger on protein graphs than on the random graphs are retained for further analysis. Figure 1 illustrates this analysis step.

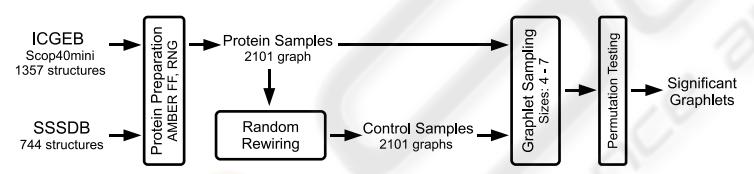

Figure 1: The graphlet analysis workflow using a random control and permutation testing.

\section{Decision Tree Analysis}

Here, we relate the extracted graphlets to global protein structure properties, e.g. the SCOP classification of each structure. Therefore, we employ decision trees as they facilitate a better understanding of the data set. Inspection of leafs and their associated samples allows relating specific graphlets to proteins and, thus, facilitates the analysis of structures with respect to interaction patterns. In addition, decision trees are native multi-class prediction algorithms and are thus a convenient choice for the prediction of various protein classes. However, decision trees tend to overfit and therefore require careful complexity regulariza-
Table 1: Results of the Graphlet Analysis.

\begin{tabular}{rrrr}
$\begin{array}{r}\text { Graphlet } \\
\text { Size }\end{array}$ & $\begin{array}{r}\text { Found } \\
\text { Graphlets }\end{array}$ & $\begin{array}{r}\text { Significant } \\
\text { Graphlets }\end{array}$ & $\begin{array}{r}\text { Filter } \\
\text { Ratio }\end{array}$ \\
\hline 4 & 427 & 22 & 0.05 \\
5 & 1,731 & 77 & 0.04 \\
6 & 5,366 & 212 & 0.04 \\
7 & 16,904 & 316 & 0.02 \\
\hline
\end{tabular}

tion.

An alternative to decision trees are neural network predictors. Neural networks represent a non-linear class of regression functions that are estimated by gradient descent. For each class a single output neuron can be integrated into the network and, thus, a single neural network can model the posterior probability of each class using a common basis of hidden neurons. However, neural networks are pure highly non-linear prediction models and, therefore, hard to interpret. Consequently, they are less suited for data mining purposes and do not provide much insight into the dataset.

Using the graphlet distributions, we encode each protein as a binary vector of graphlet occurrences. Whenever a graphlet is found for a protein, it is encoded as 1 and as 0 else. In addition, we integrate the SCOP level information from ICGEB as well as the SSSDB Motif class information as a prediction target. SCOP has four hierarchical levels: the class, fold, superfamily and family of a protein domain. Similarly, the SSSDB Motif Class encodes the super-secondary structure super class, while the SSSDB Motif Subclass denotes a more precise subdivision.

We use the partition platform from JMP (SAS Institute Inc., 2009) for learning. JMP is a reduced version of the SAS Enterprise Miner software suite, which is extensively used for professional data analysis in industry, and provides various platforms that facilitate data analysis tasks. Especially, the ability of the partition platform to easily inspect each prediction node is helpful during the analysis of the inferred graphlets. In our experiments, the Minimum Split Size (MSS) parameter is chosen to be 5, 10, 15, 20, 25 and 50, while training on graphlets of size 4 to 7 . For each model, the prediction performance is evaluated using 5-fold cross-validation to compute AUC values for each class. Then, all AUC values are averaged to yield the mean AUC (mAUC) for each combination of graphlet size and MSS. For comparison purposes, we also employ the neural nets platform.

\section{Regularization}

However, to obtain regularized decision trees, we follow the work of (Scott and Nowak, 2005). There, the 
space of decision tree hypotheses is regularized by their number of leafs and their prediction error. Let $\mathcal{T}$ be the hypothesis space of decision trees and $|T|$ denote the number of leafs of a tree $T$. Furthermore, let $n$ be the number of data samples and $\lambda$ be a weighting factor between tree complexity and prediction error, then the Complexity Regularization $C R$ for decision tree selection is given as

$$
C R=\arg \min _{T}\{\underbrace{\hat{R}}_{\text {Empirical risk }}+\lambda \underbrace{\sqrt{\frac{|T|}{n}}}_{\text {Bound on bias }}\}
$$

where $\hat{R}$ denotes the prediction error and $n$ the number of training samples. In our case, we set $\hat{R}=$ $1-m A U C$, because we are dealing with multi-class predictions and the mAUC is a performance measure between 0 and 1 that can be converted into an error by subtracting it from 1 . This error is then compared to its theoretical bound, the Uniform Deviation Bound $U D B_{n}$, which upper bounds the true risk of a decision tree and is given as

$$
U D B_{n}=\arg \min _{T \in \mathcal{T}}\left\{\hat{R}(T)+\sqrt{\frac{3|T|+\log (n)}{2 n}}\right\} .
$$

The remaining task is to find a valid $\lambda$ for regularization. We choose $\lambda$ to minimize

$$
\lambda=\arg \min _{\lambda} \sum_{T}\left(C R-U D B_{n}\right)^{2}
$$

for all considered decision trees, trained with MSS of $5,10,15,20,25$, and 50 .

\section{RESULTS \& DISCUSSION}

\section{Graphlet Extraction}

We convert 1357 proteins from the ICGEB SCOP40mini database and 744 proteins from the SSSDB into the described graphical representation. Figure 2 illustrates the result of a conversion. This results in a total of 2101 graphs left for graphlet analysis. Two other datasets from ICGEB, PCB00020 $(11,944$ structures from SCOP95) and PCB00026 (11,373 structures from CATH95), are convenient for our experiments. However, the enormous number of graphlets found in this data sets leads to infeasible computation times. Therefore, we restrict our analysis to the smallest ICGEB collection and included the SSSDB instead.

Table 1 summarizes our sampling results. It shows that increasing the graphlet pattern size also

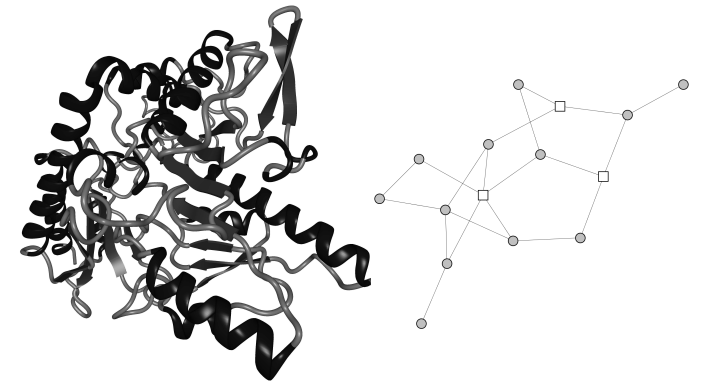

Figure 2: Structure (left) and RNG (right) of ICGEB structure d1pmma. Box nodes denote $\beta$-sheets, whereas round nodes refer to $\alpha$-helices.

increases the number of detected graphlets as well as the number of significant graphlets. However, the ratio between significant and detected graphlets remains below 5\%. Hence, we extract only a small amount of significant patterns from a large collection of graphlets (see Table 1).

\section{Decision Tree Learning}

For each target variable the decision tree minimizing $C R-U D B_{n}$ is selected as the regularized model and saved for final analysis. We find that $\lambda=1.3$ fulfills equation (5) for each target variable. We choose $\lambda$ such that the total squared deviation from the $U D B_{n}$ was minimal when averaged over all models using any size of graphlets and any number of splits. Consequently, all models are regularized and have maximal expressive power along with a reduced probability of overfitting. Table 2 lists the MSS, graphlet size, number of splits, as well as the mAUC of the final models for each target variable. We find that simple models suffice to achieve mAUC better than 0.82. In addition, at least 20 samples can be summarized in a leaf to result in a decision tree predicting one category without sacrificing prediction performance. Finally, we find that graphlets of size 5 are in most cases superior to others. Also graphlets of size 4 and 7 are used for decision tree learning and, therefore, provide useful information (Table 2).

We also compare the performance of decision trees to the performance of neural networks, also implemented in JMP. The last column of Table 2 shows the mean AUC achieved by the neural nets platform using cross-validation on a random selection of $40 \%$ of the samples, which are held out as external validation set. The platform was trained using the default values. We find that the neural network is superior to our regularized decision trees in predicting the SCOP class and SCOP family, as well as the SSSDB Motif Class. However, the performance difference does not exceed a value of 0.06 mAUC points. Con- 
Table 2: Regularized Decision Trees for each target variable.

\begin{tabular}{lccccc|c} 
Target Variable & \# classes & $\begin{array}{c}\text { Graphlet } \\
\text { Size }\end{array}$ & MSS & $\begin{array}{c}\text { Performed } \\
\text { Splits }\end{array}$ & mAUC & $\begin{array}{c}\text { Neural Net } \\
\text { mAUC }\end{array}$ \\
\hline SCOP class & 5 & 5 & 20 & 23 & 0.87 & 0.92 \\
SCOP fold & 19 & 5 & 20 & 24 & 0.87 & 0.84 \\
SCOP superfamily & 7 & 4 & 20 & 24 & 0.85 & 0.83 \\
SCOP family & 48 & 5 & 20 & 16 & 0.83 & 0.87 \\
SSSDB Motif Class & 32 & 7 & 10 & 22 & 0.93 & 0.99 \\
SSSDB Motif Subclass & 153 & 5 & 15 & 21 & 0.96 & 0.96 \\
\hline
\end{tabular}

sequently, decision trees and neural networks achieve a comparable performance and, therefore, support the descriptive value of our graphlet features.

Next, we extract all graphlets from the selected regularized decision tree models to analyse their usages with respect to all target variables. Here, we find graphlets that are used several times for the prediction of various target variables, while other graphlets are specific to one class.

While 66 graphlets are used only for the prediction of one target variable, we find two graphlets of size 5 that are used to predict 4 different target variables (Figure 3). Note that this is the maximum number achievable because two target variables are predicted using graphlets of size 4 and 7 .

In Figure 4 all SCOP superfamily graphlets are shown. Within the decision tree for the SCOP superfamily, the graphlets (a) and (b) in Figure 4 are more relevant for classification, because they are used in three and two splits, respectively. Interestingly, (a) and (b) are paths of helices containing one or two sheets, while the other graphlets consist mainly of star topologies.

Finally, we predict all detected graphlets from secondary structure sequence. Therefore, we convert each protein sequence into strings of symbols h,s, 1 encoding whether a residue is within a helix, strand or loop. We design a set of 439 features describing lengths, as well as densities, normalized to protein length, of helices, strands, loops. In addition, we design regular expressions for SSE sequence patterns, which result in binary features. Then, we train decision trees, implemented in the rpart package of $R$, and use 5-fold cross-validation using the bootstrap package to estimate the MCC (Baldi et al., 2000). We find that chains of SSEs and star topologies can be best predicted by decision trees.

\section{CONCLUSIONS}

In summary, we find that significantly overrepresented patterns in energetic SSE interactions exist and

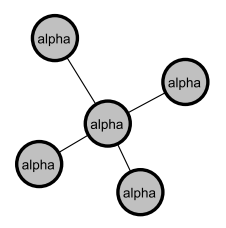

(a)

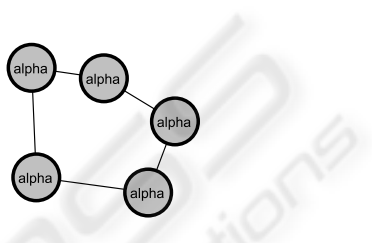

(b)
Figure 3: Graphlet 3(a) predicts 4 targets and is predictable with $\mathrm{MCC}=0.6$, while graphlet 3 (b) predicts 4 targets and is predictable with $\mathrm{MCC}=0.5$ from secondary-structure information.

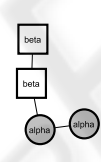

(a)
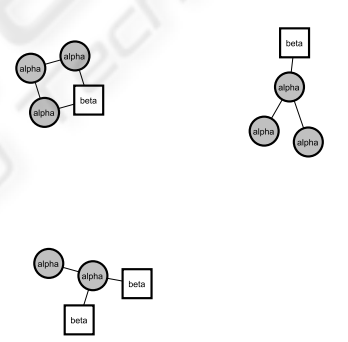

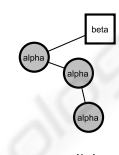

(b)
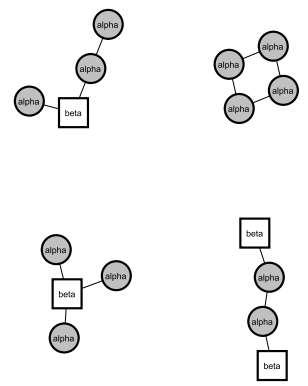

Figure 4: This figure shows all extracted graphlets used for the prediction of the SCOP superfamily.

can be found using graphlet analysis. Regularized decision tree learning on the mined patterns predicts SCOP levels and SSSDB Motifs with great accuracy (mAUC > 0.8) using about 20 graphlets. Also, the presence of a specific graphlet can be predicted from secondary structure sequence of a protein with MCC values up to 0.7 . Finally, we demonstrate that the combination of graphlet analysis using permutation testing and decision tree learning facilitates automatic categorization of protein structures.

We have shown that graphlets are predictable from the secondary structure sequence, therefore graphlets can be used as constraints for the placement of predicted secondary structure elements, when predict- 
ing the tertiary structure from the protein sequence alone. Thus, future work should focus on the usage of the predictable graphlets to improve ab initio protein structure prediction.

\section{REFERENCES}

Baldi, P., Brunak, S., Chauvin, Y., Andersen, C. A. F., and Nielsen, H. (2000). Assessing the accuracy of prediction algorithms for classification: an overview. Bioinformatics, 16(5):412-424.

Chiang, Y.-S., Gelfand, T. I., Kister, A. E., and Gelfand, I. M. (2007). New classification of supersecondary structures of sandwich-like proteins uncovers strict patterns of strand assemblage. Proteins, 68(4):915921.

Georgii, H.-O. (2004). Stochastik. de Gruyter, 2nd edition. p.198.

Holm, S. (1979). A simple sequentially rejective multiple test procedure. Scandinavian Journal of Statistics, 6(2):65-70.

Kabsch, W. and Sander, C. (1983). Dictionary of protein secondary structure: Pattern recognition of hydrogenbonded and geometrical features. Biopolymers, 22(12):2577-2637.

Kohlbacher, O. and Lenhof, H.-P. (2000). BALL-rapid software prototyping in computational molecular biology. Bioinformatics, 16(9):815-824.

Milligan, G. W. and Isaac, P. D. (1980). The validation of four ultrametric clustering algorithms. Pattern Recognition, 12(2):41 - 50 .

Milo, R., Shen-Orr, S., Itzkovitz, S., Kashtan, N., Chklovskii, D., and Alon, U. (2002). Network Motifs: Simple Building Blocks of Complex Networks. Science, 298(5594):824-827.

Murzin, A. G., Brenner, S. E., Hubbard, T., and Chothia, C. (1995). Scop: A structural classification of proteins database for the investigation of sequences and structures. Journal of Molecular Biology, 247(4):536 -540 .

Orengo, C. A., Michie, A. D., Jones, S., Jones, D. T., Swindells, M. B., and Thornton, J. M. (1997). CATHa hierarchic classification of protein domain structures. Structure, 5(8):1093-1108.

R Development Core Team (2009). R: A Language and Environment for Statistical Computing. R Foundation for Statistical Computing, Vienna, Austria. ISBN 3900051-07-0.

SAS Institute Inc. (2009). Jmp 8.0.1. www.jmp.com.

Scott, C. and Nowak, R. (2005). On the adaptive properties of decision trees. In Advances in Neural Information Processing Systems 17. MIT Press.

Sonego, P., Pacurar, M., Dhir, S., Kertesz-Farkas, A., Kocsor, A., Gaspari, Z., Leunissen, J. A. M., and Pongor, S. (2007). A Protein Classification Benchmark collection for machine learning. Nucleic Acids Res, 35(Database issue):D232-D236.
Toussaint, T., G. (1980). The relative neighbourhood graph of a finite planar set. Pattern Recognition, 12:261 268.

Vacic, V., Iakuoucheva, L., Lonardi, S., and Radivojac, P. (2010). Graphlet kernels for prediction of functional residues in protein structures. Journal of Computational Biology, 17(1):55 - 72.

Wald, A. and Wolfowitz, J. (1944). Statistical tests based on permutations of the observations. The Annals of Mathematical Statistics, 15(4):358-372.

Wassermann, L. (2004). All of statistics. Springer. theorem 14.5 . 